\title{
Data Quality-Informed Multiple Occupant Localization using Floor Vibration Sensing
}

\author{
Laixi Shi \\ laixishi@cmu.edu \\ Carnegie Mellon University
}

\author{
Yue Zhang \\ yzhang58@ucmerced.edu \\ UC Merced
}

\author{
Shijia Pan \\ span24@ucmerced.edu \\ UC Merced
}

\author{
Yuejie Chi \\ yuejiechi@cmu.edu \\ Carnegie Mellon University
}

ACM Reference Format:

Laixi Shi, Yue Zhang, Shijia Pan, and Yuejie Chi. 2020. Data Quality-Informed Multiple Occupant Localization using Floor Vibration Sensing. In Proceedings of the 21st International Workshop on Mobile Computing Systems and Applications (HotMobile '20), March 3-4, 2020, Austin, TX, USA. ACM, New York, NY, USA, 1 page. https://doi.org/10.1145/3376897.3379162

\section{DATA QUALITY-INFORMED DESIGN}

Floor vibration-based sensing technology provides an alternative approach for occupant localization, which enables various indoor applications such as elderly/child monitoring and energy usage management. Localization of multiple people is challenging due to the overlap of their footstep signals. Prior work mainly focuses on detecting the onsets of individual footsteps from overlapping signals for localization. However, due to the interference caused by the overlap, the localization error is often much higher than that of the single person case $[1,3]$.

In this work, we present a data quality-informed localization system for multiple occupant localization using vibration sensing. Figure 1 shows our proposed system. The vibration sensing module obtains floor vibration signals from the vibration sensing node array. The step-level localization module utilizes Time Difference of Arrival (TDoA) to localize each detected single step [3], where the TDoA is extracted from the onset of each footstep signal.

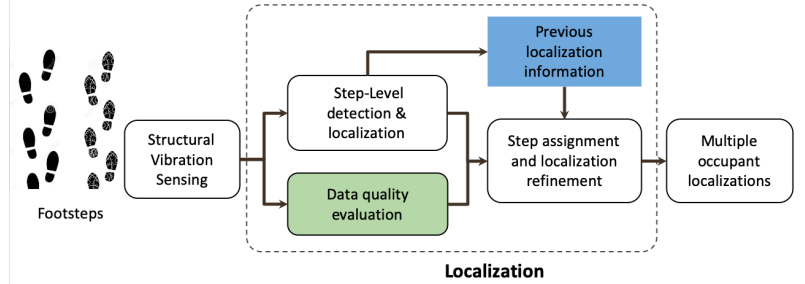

Figure 1: Overview of our proposed system.

When multiple footstep signals partially overlap, the tail of the earlier signal will distort the onset of the later arrival signal - reducing data quality and localization accuracy. When the extent of the overlap varies, the distortion to the onset estimate also varies. The data quality evaluation module quantifies this variation by ranking the overlapping condition into three different statuses gradually deteriorating: (1) clear separation; (2) visually separable; (3) visually non-separable [3], and sets different data quality parameters $r_{1}, r_{2}, r_{3}$ for these statuses respectively. The step assignment

Permission to make digital or hard copies of part or all of this work for personal or classroom use is granted without fee provided that copies are not made or distributed for profit or commercial advantage and that copies bear this notice and the full citation on the first page. Copyrights for third-party components of this work must be honored.

For all other uses, contact the owner/author(s).

HotMobile '20, March 3-4, 2020, Austin, TX, USA

(C) 2020 Copyright held by the owner/author(s).

ACM ISBN 978-1-4503-7116-2/20/03.

https://doi.org/10.1145/3376897.3379162 and localization refinement module assigns the detected step to each person based on the detection time, estimated location and history [2]. Then the location estimate is refined through a data quality-informed Kalman filter, where the data quality parameters are used as the variance of the localization report error $\sigma_{L}$ in the state-of-the-art tracking algorithm [2].

\section{EXPERIMENTAL EVALUATION}

We utilize 1000 synthetic footsteps and 14 real-world footsteps data (collected in [3]) when two persons walk in the same area simultaneously. In our localization refinement module, fixing three quality parameters $r_{1}<r_{2}<r_{3}$, the position report error $\sigma_{L}$ is set as one of them for footsteps in different overlapping conditions, for both synthetic and real-world data. We compare our method to 6 baselines without data quality prior: (1) No refinement; (2) $\sigma_{L}$ is chosen randomly in $\left(0, r_{3}\right]$; (3) $\sigma_{L}=r_{1}$; (4) $\sigma_{L}=r_{2}$; (5) $\sigma_{L}=r_{3}$; (6) $\sigma_{L}$ is chosen to make the smallest average localization error.

Figure 2 shows the localization error using synthetic data, which demonstrates that our system achieves an average localization error of just $0.42 \mathrm{~m}$, which represents $1.4 \mathrm{X}$ to $2.2 \mathrm{X}$ improvement over all the baselines. In real-world experiments, our system achieves an average localization error of just $0.46 \mathrm{~m}$, which represents $1.4 \mathrm{X}$ to 1.7X improvement over all the baseline approaches.

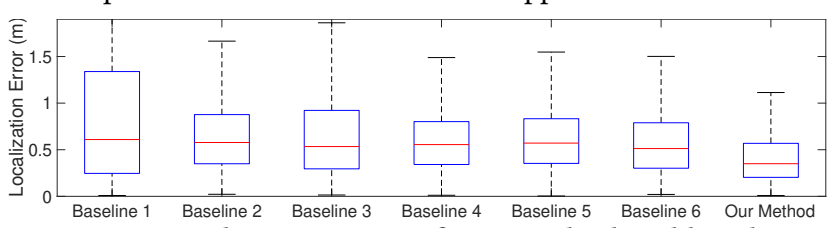

Figure 2: Localization error of our method and baselines.

\section{CONCLUSION}

In this paper, we present a data quality-informed system for multiple occupant localization through structural vibration sensing. Our system assesses data quality for detected footsteps and utilizes it as a prior for localization refinement. Our system achieves a halfmeter average localization error for the two-person case, which is comparable to the single person case.

\section{REFERENCES}

[1] Shijia Pan, Kent Lyons, Mostafa Mirshekari, Hae Young Noh, and Pei Zhang. 2016. Multiple pedestrian tracking through ambient structural vibration sensing. In Proceedings of the 14th ACM Conference on Embedded Network Sensor Systems CD-ROM. ACM, 366-367.

[2] Jeffrey D Poston. 2018. Toward Tracking Multiple Building Occupants by Footstep Vibrations. In 2018 IEEE Global Conference on Signal and Information Processing (GlobalSIP). IEEE, 86-90.

[3] Laixi Shi, Mostafa Mirshekari, Jonathon Fagert, Yuejie Chi, Hae Young Noh, Pei Zhang, and Shijia Pan. 2019. Device-free Multiple People Localization through Floor Vibration. In Proceedings of the 1st ACM International Workshop on DeviceFree Human Sensing. 57-61. 